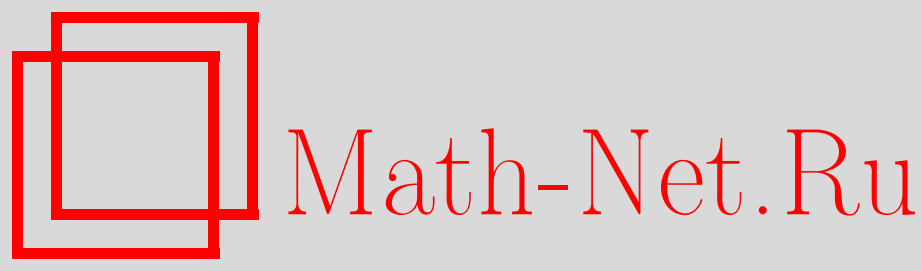

С. Ю. Вернов, Точные решения нелокальных нелинейных полевых уравнений в космологии, ТМФ, 2011, том 166, номер 3, 452-464

DOI: https://doi.org/10.4213/tmf6622

Использование Общероссийского математического портала Math-Net.Ru подразумевает, что вы прочитали и согласны с пользовательским соглашением http://www.mathnet.ru/rus/agreement

Параметры загрузки:

IP: 18.209.158.208

26 апреля 2023 г., 03:25:28

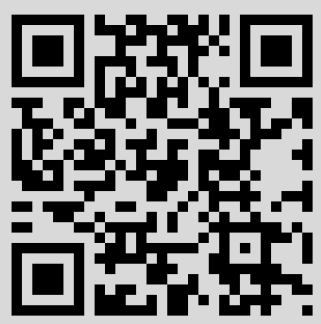




\title{
ТОЧНЫЕ РЕШЕНИЯ НЕЛОКАЛЬНЫХ НЕЛИНЕЙНЫХ ПОЛЕВЫХ УРАВНЕНИЙ В КОСМОЛОГИИ
}

\begin{abstract}
Рассматривается метод поиска точных решений уравнения для нелокального скалярного поля в неплоской метрике. В метрике Фридмана-Робертсона-Уокера предложенный метод применим для произвольного потенциала, за исключением случаев линейного и квадратичного потенциалов, и позволяет получить в квадратурах решения, зависящие от двух произвольных параметров. Точные решения найдены для произвольного кубического потенциала, рассмотрение которого мотивировано полевой теорией струн, а также для экспоненциального, логарифмического и степенного потенциалов. Показано, что добавлением скалярного поля $k$-эссенции можно получить точное решение, удовлетворяющее всем уравнениям Эйнштейна.
\end{abstract}

Ключевые слова: космология, нелокальное скалярное поле, метрика Фридмана-Робертсона-Уокера, точное решение, эллиптическая функция.

\section{1. ГРАВИТАЦИОННЫЕ МОДЕЛИ С НЕЛОКАЛЬНЫМ СКАЛЯРНЫМ ПОЛЕМ}

Нелокальные космологические модели, основанные на полевой теории струн [1], [2] и теории $p$-адических струн [3], активно развиваются [4]-[22]. Характерными особенностями нелокальных моделей являются нарушение изотропного энергетического условия и появление фантомных полей, связанных с производными высоких порядков. Локальные модели с фантомными полями считаются физически неприемлемыми из-за проблемы квантовой нестабильности [23], [24]. В работах [25]-[27] проблема нестабильности сведена к проблеме такого выбора параметров эффективной модели, чтобы нестабильность оказывалась существенной только при временах, не описываемых в рамках приближения эффективной теории. Математически это выражается в том, что приводящие к нестабильности члены рассматриваются как поправки, существенные только при малых энергиях ниже уровня физического обрезания. Данный подход позволяет рассматривать такие эффективные теории как физически приемлемые, при этом подразумевается, что эффективная теория допускает погружение в некоторую фундаментальную теорию, например полевую теорию струн.

* Научно-исследовательский институт ядерной физики им. Д.В. Скобельцына, Московский государственный университет им. М. В. Ломоносова, Москва, Россия.

E-mail: svernov@theory.sinp.msu.ru 
Интерес к космологическим моделям, связанным с полевой теорией открытых струн [4], вызван возможностью получения решений, описывающих переходы из возмущенного вакуума в истинный (так называемые роллинговые решения). После того как все массивные поля (или часть низших массивных полей) проинтегрированы с помощью уравнений движения, тахион открытой струны приобретает потенциал, имеющий нетривиальный вакуум, соответствующий минимуму энергии. В модели темной энергии [4] (см. также [28]-[30]) предполагается, что Вселенная - медленно распадающаяся $D 3$-брана, распад которой описывается модой тахиона открытой струны. Движение тахиона из нестабильного вакуума к стабильному согласно гипотезе Сена [1] описывает переход $D$-браны в истинный вакуум. Фактически получается нелокальный потенциал с параметром нелокальности, определяемым струнным масштабом. После подходящего переопределения полей потенциал можно сделать локальным, при этом кинетический член становится нелокальным. Этот нестандартный кинетический член приводит к поведению, подобному поведению фантомного поля, и может быть приближен фантомным кинетическим членом. Таким образом, поведение тахиона открытой струны эффективно моделируется скалярным полем с отрицательным кинетическим членом [31].

Из струнной теории поля также получаются асимптотические условия на решения [28]-[30]. Особый интерес представляют решения с ненулевой асимптотикой при $t \rightarrow+\infty$, ограниченные на всей действительной оси. В настоящей работе подобные решения уравнения для поля в метрике Фридмана-Робертсона-Уокера найдены для кубического и логарифмического потенциалов.

Рассмотрим гравитационную модель с нелокальным скалярным полем $\phi$, описываемую следующим действием:

$$
S=\int d^{4} x \sqrt{-g}\left(\frac{R}{16 \pi G_{\mathrm{N}}}+\frac{1}{\alpha^{\prime} g_{\mathrm{o}}^{2}}\left(\frac{1}{2} \phi \mathcal{F}\left(\alpha^{\prime} \square_{g}\right) \phi-V(\phi)\right)\right),
$$

где $G_{\mathrm{N}}$ - ньютоновская гравитационная постоянная $\left(8 \pi G_{\mathrm{N}}=1 / M_{\mathrm{P}}^{2}, M_{\mathrm{P}}-\right.$ масса Планка), $\alpha^{\prime}$ является квадратом характерной струнной длины, $g_{\text {о }}$ - константа взаимодействия открытых струн. Мы используем сигнатуру $(-,+,+,+)$, матрица $g_{\mu \nu}-$ метрический тензор, $g$ - детерминант $g_{\mu \nu}, R$ - скалярная кривизна. Потенциал $V(\phi)$ выбираем в виде произвольной дважды непрерывно дифференцируемой функции, космологическую константу считаем частью потенциала $V(\phi)$. Оператор Даламбера $\square_{g}$ действует на скалярные функции и может быть записан в следующем виде:

$$
\square_{g}=\frac{1}{\sqrt{-g}} \partial_{\mu} \sqrt{-g} g^{\mu \nu} \partial_{\nu}
$$

Скалярное поле $\phi$ является безразмерным, тогда как $\left[\alpha^{\prime}\right]=\mathrm{cm}^{2}$ и $\left[g_{\mathrm{o}}\right]=$ см. Введем безразмерные координаты $\bar{x}_{\mu}=x_{\mu} / \sqrt{\alpha^{\prime}}$, безразмерную гравитационную постоянную $\bar{G}_{N}=G_{N} / \alpha^{\prime}$ и безразмерную константу взаимодействия $\bar{g}_{\mathrm{o}}=g_{\mathrm{o}} / \sqrt{\alpha^{\prime}}$. Скаляр кривизны, вычисленный в безразмерных координатах, обозначим через $\bar{R}$, а соответствующий оператор Даламбера - через $\square_{g}$. Получаем действие $(1)$ в следующем виде:

$$
S=\int d^{4} \bar{x} \sqrt{-g}\left(\frac{\bar{R}}{16 \pi \bar{G}_{\mathrm{N}}}+\frac{1}{\bar{g}_{\mathrm{o}}^{2}}\left(\frac{1}{2} \phi \mathcal{F}\left(\bar{\square}_{g}\right) \phi-V(\phi)\right)\right),
$$


В последующих формулах мы будем использовать только безразмерные координаты и параметры, не ставя черточки над ними.

Функция $\mathcal{F}$ предполагается аналитической функцией во всей комплексной плоскости (т. е. целой функцией), поэтому она может быть представлена в виде сходящегося ряда:

$$
\mathcal{F}\left(\square_{g}\right)=\sum_{n=0}^{\infty} f_{n} \square_{g}^{n} .
$$

Из действия (1) получаем следующие уравнения:

$$
\begin{gathered}
G_{\mu \nu}=8 \pi G_{\mathrm{N}} T_{\mu \nu}, \\
\mathcal{F}\left(\square_{g}\right) \phi=\frac{d V}{d \phi},
\end{gathered}
$$

где $G_{\mu \nu}$ - тензор Эйнштейна. Тензор энергии-импульса вычисляется по стандартной формуле

$$
T_{\mu \nu}=-\frac{2}{\sqrt{-g}} \frac{\delta S}{\delta g^{\mu \nu}}=\frac{1}{g_{\mathrm{o}}^{2}}\left(E_{\mu \nu}+E_{\nu \mu}-g_{\mu \nu}\left(g^{\rho \sigma} E_{\rho \sigma}+W\right)\right)
$$

где

$$
\begin{aligned}
E_{\mu \nu} & \equiv \frac{1}{2} \sum_{n=1}^{\infty} f_{n} \sum_{l=0}^{n-1} \partial_{\mu} \square_{g}^{l} \phi \partial_{\nu} \square_{g}^{n-1-l} \phi, \\
W & \equiv \frac{1}{2} \sum_{n=2}^{\infty} f_{n} \sum_{l=1}^{n-1} \square_{g}^{l} \phi \square_{g}^{n-l} \phi-\frac{f_{0}}{2} \phi^{2}+V(\phi) .
\end{aligned}
$$

Случай квадратичного потенциала рассматривался в статьях [14], [15], [17], [19]. В работе [14] предложен алгоритм локализации уравнений Эйнштейна и найдены точные решения в пространственно плоской метрике Фридмана-Робертсона-Уокера. Данный алгоритм может быть применен только в случаях линейного или квадратичного потенциалов. В то же время из теории струн получаются потенциалы третьей и четвертой степени, а квадратичный потенциал может рассматриваться только как приближение [17]. В связи с этим активно ведется поиск решений уравнения (5) с кубическим потенциалом в метрике Фридмана-Робертсона-Уокера [16], [18]. В настоящей работе предложен метод поиска решений уравнения (5) в метрике Фридмана-Робертсона-Уокера, дающий точные частные двухпараметрические решения в замкнутом виде или в виде квадратур. Этот метод позволяет найти решения для произвольного потенциала $V(\phi)$, за исключением случаев линейного и квадратичного потенциалов. В статье подробно рассмотрен связанный с теорией струн случай кубического потенциала (раздел 3), а также случаи логарифмического, экспоненциального и степенного потенциалов (раздел 4).

Отметим, что в отличие от метода локализации [19], позволяющего локализовать все уравнения Эйнштейна, данный метод позволяет найти решения только уравнения (5), а уравнения (4) оказываются в общем случае несовместными. В разделе 5 показано, что добавлением скалярного поля $k$-эссенции удается получить точное решение всей системы нелокальных уравнений Эйнштейна. Вопрос о возможных типах дополнительной материи и возможности получения точного решения всех уравнений Эйнштейна в моделях модифицированной гравитации, например $f(R)$ гравитации, без дополнительной материи требует отдельного исследования. 
Для кубического и экспоненциального потенциалов приближенные решения уравнения (5) с обоснованным полевой теорией струн видом функции $\mathcal{F}(\square)$ найдены в статье [18], как обобщение найденных авторами решений в пространстве Минковского [32]. В отличие от работы [18], полученные в настоящей статье решения для кубического потенциала являются точными решениями уравнения (5), при этом параметр Хаббла $H(t)$ является решением уравнения, а не задается изначально.

\section{2. РЕШЕНИЯ УРАВНЕНИЯ ДВИЖЕНИЯ}

Рассмотрим нелокальное уравнение Клейна-Гордона в случае произвольного потенциала

$$
\mathcal{F}\left(\square_{g}\right) \phi=V^{\prime}(\phi),
$$

где штрих обозначает производную по $\phi$. Частным решением уравнения (8) является решение следующей системы локальных уравнений:

$$
\sum_{n=0}^{N-1} f_{n} \square_{g}^{n} \phi=V^{\prime}(\phi)-C, \quad f_{N} \square_{g}^{N} \phi=C,
$$

где $N-1$ является натуральным числом, а $C$ - произвольная константа.

Пусть $f_{1} \neq 0$, тогда можно выбрать $N=2$. Для пространственно плоской метрики Фридмана-Робертсона-Уокера, в которой интервал задается формулой

$$
d s^{2}=-d t^{2}+a^{2}(t)\left(d x_{1}^{2}+d x_{2}^{2}+d x_{3}^{2}\right),
$$

где $a(t)$ - масштабный фактор, мы получаем из (9) следующую систему:

$$
f_{1} \square_{g} \phi=-f_{1}(\ddot{\phi}+3 H \dot{\phi})=V^{\prime}(\phi)-f_{0} \phi-C, \quad f_{2} \square_{g}^{2} \phi=C,
$$

где функция $H(t)$ - параметр Хаббла: $H \equiv \dot{a}(t) / a(t)$, а точка обозначает производную по времени. Очевидно, при $f_{2}=0$ могут существовать решения только с $C=0$. Рассмотрим случай $f_{2} \neq 0$. Первое уравнение переписывается в виде

$$
H=-\frac{1}{3 \dot{\phi}}\left(\ddot{\phi}+\widetilde{V}^{\prime}(\phi)-\frac{C}{f_{1}}\right),
$$

где

$$
\widetilde{V}^{\prime}(\phi) \equiv \frac{1}{f_{1}}\left(V^{\prime}(\phi)-f_{0} \phi\right)
$$

Уравнение

$$
\left(\partial_{t}^{2}+3 H \partial_{t}\right)(\ddot{\phi}+3 H \dot{\phi})=\frac{C}{f_{2}}
$$

преобразуется к следующему виду:

$$
\left(\partial_{t}^{2}+3 H \partial_{t}\right) \widetilde{V}^{\prime}=\widetilde{V}^{\prime \prime \prime} \dot{\phi}^{2}+\widetilde{V}^{\prime \prime}(\ddot{\phi}+3 H \dot{\phi})=-\frac{C}{f_{2}} .
$$

Исключив из уравнения $H$, нетрудно получить, что

$$
\dot{\phi}^{2}=\frac{1}{\widetilde{V}^{\prime \prime \prime}}\left(\widetilde{V}^{\prime \prime} \widetilde{V}^{\prime}-\frac{C}{f_{1}} \widetilde{V}^{\prime \prime}-\frac{C}{f_{2}}\right) .
$$


Уравнение (16) решается в квадратурах. Полученное решение зависит от двух произвольных параметров $C$ и $t_{0}$, последний соответствует сдвигу по времени.

При $C=0$ получаем уравнение

$$
\dot{\phi}^{2}=\frac{\widetilde{V}^{\prime} \widetilde{V}^{\prime \prime}}{\widetilde{V}^{\prime \prime \prime}} \equiv \frac{\left(V^{\prime}-f_{0} \phi\right)\left(V^{\prime \prime}-f_{0}\right)}{f_{1} V^{\prime \prime \prime}},
$$

которое дает решения и при $f_{2}=0$.

Отметим, что предлагаемый метод неприменим в случаях линейного и квадратичного потенциалов, поскольку для них $\widetilde{V}^{\prime \prime \prime} \equiv 0$. Таким образом, данный способ поиска решений годится только для нелинейного по ф уравнения (8). В случае линейного по $\phi$ уравнения (8) поиск решений возможен с помощью метода локализации [14], [15], [19].

\section{3. КУБИЧЕСКИЙ ПОТЕНЦИАЛ}

Случай кубического потенциала активно изучается, поскольку он связан с полевой теорией бозонной струны [16], [18]. Найдем решения уравнения (8) для

$$
V(\phi)=B_{3} \phi^{3}+B_{2} \phi^{2}+B_{1} \phi+B_{0}
$$

где $B_{0}, B_{1}, B_{2}$ и $B_{3}$ - произвольные константы, при этом $B_{3} \neq 0$. Уравнение (16) имеет следующий вид:

$$
\dot{\phi}^{2}=4 C_{3} \phi^{3}+6 C_{2} \phi^{2}+4 C_{1} \phi+C_{0},
$$

где

$$
\begin{aligned}
C_{0} & =\frac{\left(B_{1}-C\right)\left(2 B_{2}-f_{0}\right)}{6 f_{1} B_{3}}-\frac{C f_{1}^{2}}{6 f_{1} f_{2} B_{3}}, & C_{2} & =\frac{2 B_{2}-f_{0}}{4 f_{1}}, \\
C_{1} & =\frac{6 B_{3}\left(B_{1}-C\right)+\left(2 B_{2}-f_{0}\right)^{2}}{24 f_{1} B_{3}}, & C_{3} & =\frac{3 B_{3}}{4 f_{1}} .
\end{aligned}
$$

Отметим, что $C_{3} \neq 0$, поскольку $B_{3} \neq 0$. Константы $B_{2}$ и $f_{0}$ входят в уравнение $(19)$ только в комбинации $2 B_{2}-f_{0}$. С помощью преобразования

$$
\phi=\frac{1}{2 C_{3}}\left(2 \xi-C_{2}\right)
$$

получаем уравнение

$$
\dot{\xi}^{2}=4 \xi^{3}-g_{2} \xi-g_{3},
$$

где

$$
\begin{aligned}
& g_{2}=3 C_{2}^{2}-4 C_{1} C_{3}=\frac{\left(2 B_{2}-f_{0}\right)^{2}-12 B_{3}\left(B_{1}-C\right)}{16 f_{1}^{2}}, \\
& g_{3}=2 C_{1} C_{2} C_{3}-C_{2}^{3}-C_{0} C_{3}^{2}=-\frac{3 B_{3} C}{32 f_{2} f_{1}}
\end{aligned}
$$

Решение уравнения (20) - это либо эллиптическая функция Вейерштрасса $\xi(t)=$ $\wp\left(t-t_{0}, g_{2}, g_{3}\right)$, где $t_{0}$ - произвольное число, либо вырожденная эллиптическая функция. Как известно [33], эллиптическая функция Вейерштрасса - это двоякопериодическая мероморфная функция, имеющая в фундаментальном параллелограмме 
периодов один двойной полюс. Если $\phi(t)$ - эллиптическая функция, то параметр Хаббла $H(t)$ - тоже эллиптическая функция.

Рассмотрим вырожденные случаи. При $g_{2}=0$ и $g_{3}=0$ решением уравнения (20) будет функция

$$
\xi=\frac{1}{\left(t-t_{0}\right)^{2}}
$$

следовательно,

$$
\phi_{1}=\frac{1}{C_{3}\left(t-t_{0}\right)^{2}}-\frac{C_{2}}{2 C_{3}}=\frac{4 f_{1}}{3 B_{3}\left(t-t_{0}\right)^{2}}-\frac{2 B_{2}-f_{0}}{6 B_{3}} .
$$

Подставляя $\phi_{1}$ в $(12)$, получаем

$$
H_{1}=\frac{5}{3\left(t-t_{0}\right)}
$$

Из условий $g_{2}=0$ и $g_{3}=0$ следует

$$
C=0, \quad B_{1}=\frac{\left(2 B_{2}-f_{0}\right)^{2}}{12 B_{3}} .
$$

Особый интерес представляют решения, ограниченные на всей действительной оси и стремящиеся к конечному пределу при $t \rightarrow \infty$. Таким решением будет функция

$$
\begin{gathered}
\phi_{2}=D_{2} \operatorname{th}\left(\beta\left(t-t_{0}\right)\right)^{2}+D_{0}, \\
D_{2}=\frac{4}{3 B_{3}} f_{1} \beta^{2}, \quad D_{0}=\frac{1}{18 B_{3}}\left(3\left(f_{0}-2 B_{2}\right)-16 f_{1} \beta^{2}\right),
\end{gathered}
$$

где $\beta$ - корень уравнения

$$
1024 f_{2} f_{1} \beta^{6}+576 f_{1}^{2} \beta^{4}+324 B_{3} B_{1}-27\left(2 B_{2}-f_{0}\right)^{2}=0 .
$$

Ограниченные решения уравнения (19) соответствуют действительным корням уравнения (26). Чисто мнимые корни уравнения (26) соответствуют неограниченным действительным решениям уравнения (19), поскольку $\operatorname{th}(\beta t)^{2}=-\operatorname{tg}(i \beta t)^{2}$. Решение $\phi_{2}$ существует при

$$
C=\frac{1}{36 B_{3}}\left(64 f_{1}^{2} \beta^{4}-3\left(2 B_{2}-f_{0}\right)^{2}+36 B_{3} B_{1}\right) .
$$

Параметр Хаббла при $t_{0}=0$ имеет вид

$$
\begin{aligned}
H_{2}= & \frac{\beta\left(2 \operatorname{ch}(\beta t)^{2}-3\right)}{3 \operatorname{ch}(\beta t) \operatorname{sh}(\beta t)}- \\
& \quad-\frac{3 B_{3}\left(D_{2} \operatorname{th}(\beta t)^{2}+D_{0}\right)^{2}+\left(2 B_{2}-f_{0}\right)\left(D_{2} \operatorname{th}(\beta t)^{2}+D_{0}\right)+B_{1}}{6 f_{1} D_{2} \beta \operatorname{th}(\beta t)\left(1-\operatorname{th}(\beta t)^{2}\right)} .
\end{aligned}
$$

Так как $t_{0}$ является произвольным комплексным число, используя равенство $\operatorname{th}(t+i \pi / 2)=\operatorname{cth} t$, получаем действительные решения:

$$
\widetilde{\phi}_{2}=D_{2} \operatorname{cth}\left(\beta\left(t-t_{0}\right)\right)^{2}+D_{0} .
$$

Отметим, что решения в терминах гиперболических функций существуют только при $C \neq 0$, поскольку $g_{3}=0$ при $C=0$, и единственным решением в терминах элементарных функций будет решение $(22)$. 


\section{4. ТОЧНЫЕ РЕШЕНИЯ ДЛЯ ДРУГИХ ВИДОВ ПОТЕНЦИАЛА}

4.1. Логарифмический потенциал. Отметим, что получить уравнение (16) в виде (19) возможно и исходя из неполиномиального потенциала. Действительно, пусть

$$
V(\phi)=C_{1} \ln (\alpha \phi),
$$

где $C_{1}$ и $\alpha$ - произвольные константы. Отметим, что константа $\alpha$ в уравнение (16) не входит:

$$
\dot{\phi}^{2}=\frac{f_{0}^{2}}{2 f_{1} C_{1}} \phi^{4}+\frac{C\left(f_{2} f_{0}-f_{1}^{2}\right)}{2 f_{1} f_{2} C_{1}} \phi^{3}+\frac{C}{2 f_{1}} \phi-\frac{C_{1}}{2 f_{1}} .
$$

При $f_{0} \neq 0$ в общем случае решениями будут эллиптические функции Якоби. При $f_{0}=0$ и $C \neq 0$ получаем частный случай уравнения (19) и решения в виде эллиптических функций Вейерштрасса.

Проанализируем действительные решения в терминах элементарных функций. Такие решения были найдены только при $f_{0}=0$.

При $C=0$ получаются решения

$$
\phi_{0}(t)=-\frac{\sqrt{-2 C_{1} f_{1}}}{2 f_{1}}\left(t-t_{0}\right), \quad H_{0}=\frac{2}{3\left(t-t_{0}\right)}+\frac{f_{0}}{3 f_{1}}\left(t-t_{0}\right) .
$$

При $C \neq 0$ существует решение

$$
\phi_{\ln }=\widetilde{D}_{2} \operatorname{th}^{2}\left(A\left(t-t_{0}\right)\right)+\widetilde{D}_{0},
$$

где $A$ - произвольное число,

$$
\widetilde{D}_{2}=-\frac{C_{1}\left(32 f_{2} A^{2}-9 f_{1}\right)}{18 A f_{1}\left(16 f_{2} A^{2}-3 f_{1}\right) \varsigma}, \quad \widetilde{D}_{0}=\frac{C_{1}}{12 A f_{1} \varsigma}, \quad \varsigma= \pm \sqrt{\frac{C_{1}}{144 f_{2} A^{2}-27 f_{1}}},
$$

при этом $C=64 A^{3} f_{2} \varsigma$. Данному решению при $t_{0}=0$ соответствует параметр Хаббла

$$
H_{\ln }=\frac{A\left(2 \operatorname{ch}(A t)^{2}-3\right)}{3 \operatorname{ch}(A t) \operatorname{sh}(A t)}-\frac{C_{1} \operatorname{ch}(A t)^{2}}{6 A f_{1} \widetilde{D}_{2}\left(\widetilde{D}_{2} \operatorname{th}(A t)^{2}+\widetilde{D}_{0}\right) \operatorname{th}(A t)} .
$$

4.2. Экспоненциальный потенциал. В работе [18] наряду с кубическим был рассмотрен экспоненциальный потенциал и были найдены приближенные решения уравнения (8).

Пусть $V(\phi)=C_{1} e^{\alpha \phi}$. При $f_{0}=0$ и $C=0$ решения системы (9) получаются в терминах элементарных функций и имеют следующий вид:

$$
\phi_{\exp }(t)=\frac{1}{\alpha} \ln \left(\frac{4 f_{1}}{C_{1} \alpha^{2}\left(t-t_{0}\right)^{2}}\right), \quad H_{\exp }(t)=\frac{1}{t-t_{0}} .
$$

Отметим, что полученный вид параметра Хаббла пропорционален параметру Хаббла, использованному в работе [18], и параметру Хаббла, получаемому в случае кубического потенциала (формула (23)). 
4.3. Степенной потенциал. Рассмотрим решения в случае потенциала $V(\phi)=$ $C_{1} \phi^{n}$. При $f_{0}=0$ уравнение (16) примет вид

$$
\dot{\phi}^{2}=\frac{C_{1}^{2} f_{2} n^{2}(n-1) \phi^{n}-C_{1} C f_{2} n(n-1) \phi-C f_{1}^{2} \phi^{3-n}}{f_{1} f_{2} C_{1} n(n-1)(n-2)} .
$$

При $C=0$ данное уравнение сводится к

$$
\dot{\phi}^{2}=\frac{C_{1} n \phi^{n}}{f_{1}(n-2)}
$$

и имеет решение в виде элементарной функции:

$$
\phi_{n}(t)=2^{2 /(n-2)}\left(\frac{f_{1}}{C_{1} n(n-2)\left(t-t_{0}\right)^{2}}\right)^{1 /(n-2)} .
$$

Соответствующий параметр Хаббла равен

$$
H_{n}(t)=\frac{3 n-4}{3(n-2)\left(t-t_{0}\right)} .
$$

При $n=4 / 3$ получаем частное решение уравнения (8) в пространстве Минковского:

$$
\phi_{\mathrm{M}}(t)= \pm \frac{2 C_{1} \sqrt{-2 f_{1} C_{1}}}{27 f_{1}^{2}}\left(t-t_{0}\right)^{3} .
$$

Отметим, что в пространстве Минковского точные, ограниченные на всей действительной оси решения для нелокальных уравнений со степенными потенциалами найдены в статье [29].

\section{5. КОСМОЛОГИЧЕСКАЯ МОДЕЛЬ С НЕЛОКАЛЬНЫМ СКАЛЯРНЫМ ПОЛЕМ И ПОЛЕМ $k$-ЭССЕНЦИИ}

Цель данного раздела - показать, что добавлением скалярного поля $k$-эссенции $\Psi$ можно получить систему уравнений Эйнштейна, имеющую точное решение, при этом параметр Хаббла и нелокальное поле заданы формулами (12) и (16) соответственно. Модели $k$-эссенции рассматриваются в космологии и как модели инфляции [34]-[36], и как модели темной энергии [37]-[40], [27] (см. также работу [41] и ссылки в ней).

Рассмотрим следующее действие:

$$
S_{2}=\int d^{4} x \sqrt{-g}\left(\frac{R}{16 \pi G_{\mathrm{N}}}+\frac{1}{g_{\mathrm{o}}^{2}}\left(\frac{1}{2} \phi \mathcal{F}\left(\square_{g}\right) \phi-V(\phi)\right)-\mathcal{P}(\Psi, X)\right),
$$

где $X \equiv-g^{\mu \nu} \partial_{\mu} \Psi \partial_{\nu} \Psi$. В метрике Фридмана-Робертсона-Уокера функция $\Psi$ зависит только от времени, поэтому $X=\dot{\Psi}^{2}$.

Следуя работе [27], выберем давление в виде

$$
\mathcal{P}(\Psi, X)=\frac{1}{2}\left(p_{q}(\Psi)-\varrho_{q}(\Psi)\right)+\frac{1}{2}\left(p_{q}(\Psi)+\varrho_{q}(\Psi)\right) X+\frac{1}{2} M^{4}(\Psi)(X-1)^{2} .
$$


Функции $p_{q}(\Psi), \varrho_{q}(\Psi)$ и $M^{4}(\Psi)$ будем считать произвольными дифференцируемыми функциями. Плотность энергии поля $k$-эссенции равна

$$
\mathcal{E}(\Psi, X)=\left(p_{q}(\Psi)+\varrho_{q}(\Psi)\right) X+2 M^{4}(\Psi)\left(X^{2}-X\right)-\mathcal{P}(\Psi, X) .
$$

Уравнения Эйнштейна, полученные варьированием $S_{2}$, имеют следующий вид:

$$
\begin{aligned}
3 H^{2} & =8 \pi G_{\mathrm{N}}(\varrho+\mathcal{E}), \\
2 \dot{H}+3 H^{2} & =-8 \pi G_{\mathrm{N}}(p+\mathcal{P}) .
\end{aligned}
$$

Варьированием $S_{2}$ также получаются уравнение $(8)$ и уравнение для поля $k$-эссенции $\Psi$

$$
\dot{\mathcal{E}}=-3 H(\mathcal{E}+\mathcal{P})
$$

В метрике Фридмана-Робертсона-Уокера тензор энергии-импульса (6) имеет вид

$$
T_{\mu \nu}=g_{\mu \nu} \operatorname{diag}(-\varrho, p, p, p),
$$

где

$$
\begin{aligned}
& \varrho=\frac{1}{g_{\mathrm{o}}^{2}}\left(\sum_{n=1}^{\infty} \frac{f_{n}}{2}\left(\sum_{l=0}^{n-1} \partial_{t} \square^{l} \phi \partial_{t} \square^{n-1-l} \phi+\sum_{l=1}^{n-1} \square^{l} \phi \square^{n-l} \phi\right)-\frac{f_{0}}{2} \phi^{2}+V(\phi)\right), \\
& p=\frac{1}{g_{\mathrm{o}}^{2}}\left(\sum_{n=1}^{\infty} \frac{f_{n}}{2}\left(\sum_{l=0}^{n-1} \partial_{t} \square^{l} \phi \partial_{t} \square^{n-1-l} \phi-\sum_{l=1}^{n-1} \square^{l} \phi \square^{n-l} \phi\right)+\frac{f_{0}}{2} \phi^{2}-V(\phi)\right) .
\end{aligned}
$$

Пусть $\phi_{2}-$ решение системы (9) при $N=2$. Используя $\square_{g}^{2} \phi_{2}=C / f_{2}$, получаем

$$
\varrho\left(\phi_{2}\right)=E_{00}\left(\phi_{2}\right)+W\left(\phi_{2}\right), \quad p\left(\phi_{2}\right)=E_{00}\left(\phi_{2}\right)-W\left(\phi_{2}\right),
$$

где

$$
\begin{aligned}
E_{00}\left(\phi_{2}\right) & =\frac{1}{2 g_{\mathrm{o}}^{2}}\left(f_{1}\left(\partial_{t} \phi_{2}\right)^{2}+2 f_{2} \partial_{t} \phi_{2} \partial_{t} \square_{g} \phi_{2}+f_{3}\left(\partial_{t} \square_{g} \phi_{2}\right)^{2}\right), \\
W\left(\phi_{2}\right) & =\frac{1}{g_{\mathrm{o}}^{2}}\left(\frac{f_{2}}{2}\left(\square_{g} \phi_{2}\right)^{2}+\frac{f_{3} C}{f_{2}} \square_{g} \phi_{2}+\frac{f_{4} C^{2}}{2 f_{2}^{2}}-\frac{f_{0}}{2} \phi_{2}^{2}+V\left(\phi_{2}\right)\right) .
\end{aligned}
$$

Модели $k$-эссенции (без дополнительных полей) обладают одним полезным свойством. Для любой действительной дифференцируемой функции $H_{0}(t)$ существуют такие действительные дифференцируемые функции $\varrho_{q}(\Psi)$ и $p_{q}(\Psi)$, что функции $H_{0}\left(t-t_{0}\right)$ и $\Psi(t)=t-t_{0}$ являются частным решением системы (42), (43). Данное свойство можно обобщить и на случай моделей с дополнительным нелокальным скалярным полем, описываемых действием (39). В самом деле, при $\Psi(t)=t-t_{0}$

$$
\mathcal{E}=\varrho_{q}(\Psi)=\varrho_{q}\left(t-t_{0}\right), \quad \mathcal{P}=p_{q}(\Psi)=p_{q}\left(t-t_{0}\right) .
$$

Подставляя в $(42)$ выражения для $\varrho_{q}$ и $p_{q}$, получаем

$$
\begin{gathered}
\varrho_{q}(\Psi)=\varrho_{q}\left(t-t_{0}\right)=\frac{3}{8 \pi G_{\mathrm{N}}} H^{2}\left(t-t_{0}\right)-\varrho\left(t-t_{0}\right), \\
p_{q}(\Psi)=p_{q}\left(t-t_{0}\right)=-\varrho_{q}\left(t-t_{0}\right)-\varrho\left(t-t_{0}\right)-p\left(t-t_{0}\right)-\frac{1}{4 \pi G_{\mathrm{N}}} \dot{H}\left(t-t_{0}\right) .
\end{gathered}
$$


Как легко видеть, система (42), (43) и уравнение (8) имеют точное частное решение, при этом функции $H\left(t-t_{0}\right)$ и $\phi\left(t-t_{0}\right)$ - полученное решение уравнения $(8)$, а $\Psi(t)=t-t_{0}$.

Таким образом, алгоритм получения точных решений следующий: для заданного потенциала $V(\phi)$ находим $H(t)$ и $\phi(t)$, вычисляем тензор энергии-импульса и подставляем полученные значения в (47). Полученные значения $\varrho_{q}$ и $p_{q}$ дадут точно решаемую модель с нелокальным скалярным полем и полем $k$-эссенции. Функция $M(\Psi)$ может быть выбрана произвольно.

Проиллюстрируем данную схему на простейшем примере, связанном с кубическим потенциалом. Рассмотрим решения (22), (23). Условия (24) существования данного решения оставляют произвольными константы $B_{3}$ и $B_{2}$. Пользуясь произволом $B_{2}$, мы можем, не ограничивая общности, положить $f_{0}=0$. Также для компактности записи мы положим $t_{0}=0$. Для решения $(22),(23)$ получаем

$$
\square_{g} \phi_{1}=\frac{16 f_{1}}{3 B_{3} t^{4}}
$$

следовательно,

$$
E_{00}=\frac{32 f_{1}^{2}\left(f_{1} t^{4}+16 f_{2} t^{2}+64 f_{3}\right)}{9 B_{3}^{2} t^{10}}, \quad W=\frac{128 f_{2} f_{1}^{2}}{9 g_{\mathrm{o}}^{2} B_{3}^{2} t^{8}}+\frac{1}{g_{\mathrm{o}}^{2}} V\left(\phi_{1}\right) .
$$

В итоге получаем

$$
\begin{aligned}
& \varrho_{q}(\Psi)=\frac{B_{2}^{3}-27 B_{0} B_{3}^{2}}{27 g_{\mathrm{o}}^{2} B_{3}^{2}}+\frac{25}{24 \pi G_{\mathrm{N}} \Psi^{2}}-\frac{160 f_{1}^{3}}{27 g_{\mathrm{o}}^{2} B_{3}^{2} \Psi^{6}}-\frac{640 f_{2} f_{1}^{2}}{9 g_{\mathrm{o}}^{2} B_{3}^{2} \Psi^{8}}-\frac{2048 f_{3} f_{1}^{2}}{9 g_{\mathrm{o}}^{2} B_{3}^{2} \Psi^{10}}, \\
& p_{q}(\Psi)=-\frac{B_{2}^{3}-27 B_{0} B_{3}^{2}}{27 g_{\mathrm{o}}^{2} B_{3}^{2}}-\frac{5}{8 \pi G_{\mathrm{N}} \Psi^{2}}-\frac{32 f_{1}^{3}}{27 g_{\mathrm{o}}^{2} B_{3}^{2} \Psi^{6}}-\frac{128 f_{2} f_{1}^{2}}{3 g_{\mathrm{o}}^{2} B_{3}^{2} \Psi^{8}}-\frac{2048 f_{3} f_{1}^{2}}{9 g_{\mathrm{o}}^{2} B_{3}^{2} \Psi^{10}}
\end{aligned}
$$

Таким образом, можно сделать вывод, что добавление поля $k$-эссенции позволяет построить для найденного решения уравнения (8) самосогласованную систему уравнений Эйнштейна. Выбор скалярного поля $k$-эссенции в качестве дополнительного поля продиктован стремлением получить самосогласованную систему уравнений Эйнштейна без ограничений на вид потенциала и решения.

\section{6. ЗАКЛЮЧЕНИЕ}

В настоящей статье предложен метод поиска точных решений уравнения (8) в метрике Фридмана-Робертсона-Уокера. Показано, что для произвольного потенциала нелокального скалярного поля, отличного от линейного и квадратичного, существует частное двухпараметрическое решение, которое может быть найдено в квадратурах.

Подробно рассмотрен случай потенциала третьей степени, возникающий в моделях, связанных с полевой теорией струн. Показано, что решениями будут либо эллиптические функции Вейерштрасса, либо их вырожденные случаи. Например, было получено решение, выражаемое в терминах гиперболического тангенса. Также найдены точные решения в терминах элементарных функций для логарифмического, экспоненциального и степенного потенциалов. 
Отметим, что данный метод позволяет найти решения только уравнения для поля, а не всей системы уравнений Эйнштейна. Показано, что добавление скалярного поля $k$-эссенции позволяет построить для найденного решения уравнения (8) самосогласованную систему уравнений Эйнштейна. Локализация системы уравнений Эйнштейна в космологических моделях с нелокальным скалярным полем и произвольным потенциалом является актуальной задачей и требует отдельного исследования ${ }^{1)}$.

Благодарности. Автор выражает благодарность организаторам Международной Боголюбовской конференции (Москва-Дубна, Россия, 20-26 августа 2009 г.) за возможность представить результаты своей работы и финансовую поддержку. Автор благодарит И. Я. Арефьеву и Н. Нунеша за полезные и стимулирующие обсуждения. Работа частично поддержана РФФИ (грант № 08-01-00798) и Программой поддержки ведущих научных школ (грант НШ-4142.2010.2), а также Российским федеральным агентством по науке и инновациям (государственные контракты 02.740.11.5057 и 02.740.11.0244).

\section{Список литературы}

[1] K. Ohmori, A review on tachyon condensation in open string field theories, arXiv: hep-th/0102085; I. Ya. Aref'eva, D. M. Belov, A. A. Giryavets, A.S. Koshelev, P. B. Medvedev, "Noncommutative field theories and (super)string field theories", Particles and Fields, eds. G. A. Alves, O. J. P. Éboli, V. O. Rivelles, World Sci., River Edge, NJ, 2002, 1-163, arXiv: hep-th/0111208; W. Taylor, "Lectures on D-branes, tachyon condensation, and string field theory", Lectures on Quantum Gravity, Ser. Cent. Estud. Cient., eds. A. Gomberoff, D. Marolf, Springer, New York, 2005, 151-206, arXiv: hep-th/0301094.

[2] И. Я. Арефьева, ТМФ, 163:3 (2010), 355-365.

[3] L. Brekke, P. G. O. Freund, M. Olson, E. Witten, Nucl. Phys. B, 302:3 (1988), 365-402; P. H. Frampton, Y. Okada, Phys. Rev. D, 37:10 (1988), 3077-3079; V.S. Vladimirov, I. V. Volovich, E. I. Zelenov, p-Adic Analysis and Mathematical Physics, Ser. Sov. East European Math., 10, World Sci., Singapore, 1994; B. Dragovich, A. Yu. Khrennikov, S. V. Kozyrev, I. V. Volovich, Anal. Appl., 1:1 (2009), 1-17, arXiv: 0904.4205.

[4] I. Ya. Aref'eva, "Nonlocal string tachyon as a model for cosmological dark energy", p-Adic Mathematical Physics, AIP Conf. Proc., 826, eds. A. Yu. Khrennikov, Z. Rakić, I. V. Volovich, AIP, Melville, NY, 2006, 301-311, arXiv: astro-ph/0410443; "Stringy model of cosmological dark energy", Particles, Strings, and Cosmology, AIP Conf. Proc., 957, eds. A. Rajantie, P. Dauncey, C. Contaldi, H. Stoica, AIP, Melville, NY, 2007, 297-300, arXiv: 0710.3017.

[5] G. Calcagni, JHEP, 05 (2006), 012, 25 pp., arXiv: hep-th/0512259.

[6] N. Barnaby, T. Biswas, J. M. Cline, JHEP, 04 (2007), 056, 35 pp., arXiv: hep-th/0612230; N. Barnaby, J. M. Cline, J. Cosmol. Astropart. Phys., 07 (2007), 017, 30 pp., arXiv: 0704.3426; N. Barnaby, Can. J. Phys., 87:3 (2009), 189-194, arXiv: 0811.0814.

[7] A. S. Koshelev, JHEP, 04 (2007), 029, 19 pp., arXiv: hep-th/0701103.

[8] I. Ya. Aref'eva, L. V. Joukovskaya, S. Yu. Vernov, JHEP, 07 (2007), 087, 36 pp., arXiv: hep-th/0701184.

[9] I. Ya. Aref'eva, I. V. Volovich, Int. J. Geom. Methods Mod. Phys., 4:5 (2007), 881-895, arXiv: hep-th/0701284.

[10] J. E. Lidsey, Phys. Rev. D, 76:4 (2007), 043511, 5 pp., arXiv: hep-th/0703007.

\footnotetext{
1) Метод локализации построен только в случае линейного или квадратичного потенциала [19].
} 
[11] G. Calcagni, M. Montobbio, G. Nardelli, Phys. Rev. D, 76:12 (2007), 126001, 20 pp., arXiv: 0705.3043; G. Calcagni, G. Nardelli, Phys. Rev. D, 78:12 (2008), 126010, 20 pp., arXiv: 0708.0366; G. Calcagni, M. Montobbio, G. Nardelli, Phys. Lett. B, 662:3 (2008), 285-289, arXiv: 0712.2237; G. Calcagni, G. Nardelli, Phys. Lett. B, 669:1 (2008), 102-106, arXiv: 0802.4395.

[12] L. V. Joukovskaya, Phys. Rev. D, 76:10 (2007), 105007, 12 pp., arXiv: 0707.1545; "Rolling Tachyon in nonlocal cosmology", Particles, Strings, and Cosmology, AIP Conf. Proc., 957, eds. A. Rajantie, P. Dauncey, C. Contaldi, H. Stoica, AIP, Melville, NY, 2007, 325-328, arXiv: 0710.0404.

[13] N. Barnaby, N. Kamran, JHEP, 02 (2008), 008, 39 pp., arXiv: 0709.3968; JHEP, 12 (2008), 022, 27 pp., arXiv: 0809.4513.

[14] I. Ya. Aref'eva, L. V. Joukovskaya, S. Yu. Vernov, J. Phys. A, 41:30 (2008), 304003, 14 pp., arXiv: 0711.1364 .

[15] D. J. Mulryne, N. J. Nunes, Phys. Rev. D, 78:6 (2008), 063519, 16 pp., arXiv: 0805.0449; "Non-linear non-local Cosmology", The Dark Side of the Universe, 4th International Workshop on the Dark Side of the Universe, AIP Conf. Proc., 1115, ed. Kh. Shaaban, Springer, Berlin, 2009, 329-334, arXiv: 0810.5471.

[16] L. V. Joukovskaya, JHEP, 02 (2009), 045, 18 pp., arXiv: 0807.2065.

[17] A. S. Koshelev, S. Yu. Vernov, Cosmological perturbations in SFT inspired non-local scalar field models, arXiv: 0903.5176.

[18] G. Calcagni, G. Nardelli, Internat. J. Modern Phys. D, 19:3 (2010), 329-338, arXiv: 0904.4245.

[19] S. Yu. Vernov, Class. Quant. Grav., 27:3 (2010), 035006, 16 pp., arXiv: 0907.0468; Localization of the SFT inspired nonlocal linear models and exact solutions; Писвма в ЭЧАЯ, 8:3 (2011), в печати; arXiv: 1005.0372.

[20] G. Calcagni, G. Nardelli, Phys. Rev. D, 82:12 (2010), 123518, 17 pp., arXiv: 1004.5144.

[21] A. S. Koshelev, S. Yu. Vernov, Analysis of scalar perturbations in cosmological models with a non-local scalar field, arXiv: 1009.0746.

[22] Ф. Галли, А. С. Кошелев, ТМФ, 164:3 (2010), 401-409, arXiv: 1010.1773.

[23] И. Я. Арефьева, И. В. Волович, ТМФ, 155:1 (2008), 3-12, arXiv: hep-th/0612098.

[24] R. Kallosh, J. U. Kang, A. Linde, V. Mukhanov, J. Cosmol. Astropart. Phys., 04 (2008), 018, 23 pp., arXiv: 0712.2040.

[25] S. Weinberg, Phys. Rev. D, 77:12 (2008), 123541, 7 pp., arXiv: 0804.4291.

[26] J.Z. Simon, Phys. Rev. D, 41:12 (1990), 3720-3733.

[27] P. Creminelli, G. D’Amico, J. Noreña, F. Vernizzi, J. Cosmol. Astropart. Phys., 02 (2009), 018, 36 pp., arXiv: 0811.0827.

[28] И. Я. Арефьева, С. Ю. Вернов, А. С. Кошелев, ТМФ, 148:1 (2006), 23-41, arXiv: astro-ph/0412619; I. Ya. Aref'eva, A. S. Koshelev, S. Yu. Vernov, Phys. Lett. B, 628:1-2 (2005), 1-10, arXiv: astro-ph/0505605; Phys. Rev. D, 72:6 (2005), 064017, 11 pp., arXiv: astro-ph/0507067.

[29] I. Ya. Aref'eva, L. V. Joukovskaya, JHEP, 10 (2005), 087, 23 pp., arXiv: hep-th/0504200.

[30] С. Ю. Вернов, ТМФ, 155:1 (2008), 47-61, arXiv: astro-ph/0612487.

[31] I. Ya. Aref'eva, A. S. Koshelev, L. V. Joukovskaya, JHEP, 09 (2003), 012, 15 pp., arXiv: hep-th/0301137.

[32] G. Calcagni, G. Nardelli, Nucl. Phys. B, 823:1-2 (2009), 234-253, arXiv: 0904.3744.

[33] Г. Бейтмен, А. Эрдейи, Высшие трансиендентные функиии. Эллиптические и автоморфные функиии. Функиии Ламе и Матъе, т. 3, Справочная математическая библиотека, Наука, М., 1967.

[34] C. Armendáriz-Picón, T. Damour, V. Mukhanov, Phys. Lett. B, 458:2-3 (1999), 209-218, arXiv: hep-th/9904075; J. Garriga, V.F. Mukhanov, Phys. Lett. B, 458:2-3 (1999), 219-225; arXiv: hep-th/9904176. 
[35] N. Arkani-Hamed, H.-C. Cheng, M. A. Luty, S. Mukohyama, JHEP, 05 (2004), 074, 36 pp., arXiv: hep-th/0312099; N. Arkani-Hamed, P. Creminelli, Sh. Mukohyama, M. Zaldarriaga, J. Cosmol. Astropart. Phys., 04 (2004), 001, 18 pp., arXiv: hep-th/0312100.

[36] P. Creminelli, M. A. Luty, Al. Nicolis, L. Senatore, JHEP, 12 (2006), 080, 30 pp., arXiv: hep-th/0606090.

[37] C. Armendariz-Picon, V. Mukhanov, P. J. Steinhardt, Phys. Rev. Lett., 85:21 (2000), 4438-4441, arXiv: astro-ph/0004134; Phys. Rev. D, 63:10 (2001), 103510, 13 pp., arXiv: astro-ph/0006373.

[38] A. D. Rendall, Class. Quant. Grav., 23:5 (2006), 1557-1569, arXiv: gr-qc/0511158.

[39] J. U. Kang, V. Vanchurin, S. Winitzki, Phys. Rev. D, 76:8 (2007), 083511, 27 pp., arXiv: 0706.3994.

[40] E. Babichev, V. Mukhanov, A. Vikman, JHEP, 02 (2008), 101, 45 pp., arXiv: 0708.0561.

[41] A. Vikman, $k$-essence: cosmology, causality and emergent geometry, Ph. D. Thesis, LMU, Munich, 2007, 108 pp., edoc.ub.uni-muenchen.de/7761.

Поступила в редакцию 18.05.2010, после доработки 4.10.2010 